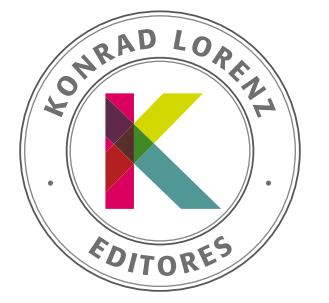

\title{
Análisis de las competencias en segundo idioma en los programas de psicología colombianos
}

\author{
Julio César Alonso C. ${ }^{\mathrm{a}}$, Daniela Estrada ${ }^{\mathrm{b}}$, Brigitte Vanessa Mueces ${ }^{\mathrm{b}}$ \\ y Marithza Sandoval-Escobar ${ }^{c}$
}

\author{
a Departamento de Economía, Universidad Icesi, Cali, Colombia \\ ${ }^{b}$ Centro de Investigación en Economía y Finanzas, Universidad Icesi, Cali, Colombia \\ ' Fundación Universitaria Konrad Lorenz, Bogotá, Colombia
}

Recibido el 5 de junio de 2018; aceptado el 23 de octubre de 2018

\author{
PALABRAS CLAVE \\ competencias \\ del psicólogo, \\ proyecto Tuning, \\ bilingüismo, \\ segundo idioma, \\ Saber Pro
}

\begin{abstract}
Resumen En este artículo se identificó el dominio del inglés de los futuros egresados de los programas de psicología colombianos. Para tal fin, se analizaron los resultados del componente en inglés del Examen de Estado de Calidad de la Educación Superior (Saber Pro) presentado por los futuros egresados de los programas de psicología de las instituciones de educación superior (IES) entre el 2011 y 2016, en los tres tipos de modalidades que existen: presencial, virtual y a distancia, y teniendo en cuenta si el programa se encontraba o no acreditado. Los resultados muestran que, de los 102 programas, solo 6 cumplieron en el 2014 con la meta establecida por el Gobierno colombiano del 20\% de sus egresados en nivel pre-avanzado o superior para ese año. Todos corresponden a la modalidad presencial y de los 30 programas que estaban acreditados en dicho año, solo el $13 \%$ cumple con la meta establecida. Además, se discute el cumplimiento de la meta para los demás años. Se discuten los resultados a la luz de las metas establecidas por el Gobierno Nacional respecto al nivel de inglés de los egresados y de las competencias comunicativas establecidas para los psicólogos en el proyecto Tuning, entre otros.

(C) 2018 Fundación Universitaria Konrad Lorenz. Este es un artículo Open Access bajo la licencia CC BY-NC-ND (http://creativecommons.org/licenses/bync-nd/4.0/).
\end{abstract}

* Autor para correspondencia.

Correo electrónico: jcalonso@icesi.edu.co

http://dx.doi.org/10.14349/sumapsi.2018.v25.n2.4

0120-0534/@ 2018 Fundación Universitaria Konrad Lorenz. Este es un artículo Open Access bajo la licencia CC BY-NC-ND (http://creativecommons.org/licenses/by-nc-nd/4.0/). 


\author{
KEYWORDS \\ psychologist \\ competences, \\ Tuning project, \\ bilingualism, \\ second language, \\ Saber Pro
}

\section{Analysis of second language competences in Colombian psychology programs}

Abstract This study aimed to identify the English proficiency of future graduates of Colombian psychology programs. To this end, we analyze the results of the mandatory English component of the State Examination of Quality of Higher Education (Saber Pro) presented by future graduates of the psychology programs between 2011 and 2016. The document also compares the results by the delivery method (face-to-face, virtual and distance) and national accreditation. The Colombian Government set the exit target that $20 \%$ of undergraduates should reach at least pre-advance by 2014. The results show that, of the 102 programs, only 6 met the goal in 2014 . All programs that reached the target used a face-to-face delivery method. Results also show that $13 \%$ of the accredited 30 programs in that year met the goal. The results are discussed in light of the goals established by the national government regarding the level of graduate English and the communication skills set for the psychologists in the Tuning project, among others.

(C) 2018 Fundación Universitaria Konrad Lorenz. This is an open access article under the CC BY-NCND license (http://creativecommons.org/licenses/bync-nd/4.0/).
El Gobierno colombiano es consciente de la necesidad de tener una fuerza laboral competente en el manejo de una lengua extranjera como el inglés. Existe evidencia que una fuerza laboral competente en inglés promueve la productividad, el intercambio comercial y el crecimiento económico de los países (Fidrmuc \& Fidrmuc, 2014; Ku \& Zussmanb, 2010; Lee, 2012). Es por eso por lo que desde hace más de 40 años, el Gobierno colombiano ha promovido, mediante el Ministerio de Educación Nacional (MEN), la formación de los estudiantes y profesores en inglés. El plan más ambicioso propuesto por el MEN se estableció en 2005 y se llamó Programa Nacional de Bilingüismo Colombia (PNB) 2004-2019 (MEN, 2014). En este se propusieron metas del nivel de inglés que deberían alcanzar los graduados de bachillerato y de programas profesionales universitarios en 2019. Asimismo, se establecieron metas intermedias para 2014: 40\% de los estudiantes de secundaria deberían alcanzar un nivel pre-intermedio y $20 \%$ de los graduados, de educación superior de carácter universitario, debían clasificar en nivel intermedio o superior en inglés (Alonso, Casasbuenas, Gallo \& Torres, 2012).

En contraste, el estudio de Sánchez (2013) muestra que al analizar históricamente el desarrollo del conocimiento del idioma inglés hasta 2011, los resultados han sido bajos, a pesar de las metas y programas del Gobierno Nacional. Así, el $90 \%$ de los estudiantes de educación media alcanzan un A1; mientras que en la educación superior, dicha proporción es del 60\%; solo el 2\% de los bachilleres alcanzó el nivel B1 y en la educación superior el 6,5\% el nivel B+.

Igualmente, en el seguimiento realizado por el MEN respecto a los resultados en educación superior, en cuanto a la vinculación laboral de 2014 de los graduados de 2013, se encontró que el 57,6\% de los recién graduados manifiestan que dominan el inglés como su segundo idioma; sin embargo, su percepción de manejo se concentra en el nivel medio (MEN, 2015). El 40\% de los estudiantes reportaron un dominio precario del idioma inglés como segunda lengua.

En virtud de estos resultados, se han adelantado estudios para establecer el dominio de la competencia en potenciales graduados de distintas disciplinas, de forma que las instituciones y comunidades académicas puedan obtener una retroalimentación acerca de la formación en segunda lengua, que les permita implementar acciones de me- joramiento al interior del currículo y de sus programas de internacionalización. Algunos de los estudios disponibles se han realizado para los graduandos de ingeniería de sistemas (Alonso, Estrada y Martínez, 2016), en programas de economía (Alonso, Estrada \& Mueces, 2018) y los graduandos de medicina (Alonso, Díaz, Estrada \& Mueces, 2018). Aunque para todas las áreas del conocimiento es importante dominar el inglés y evaluar si cumplieron o no la meta, este artículo se concentra en analizar el dominio del inglés de los potenciales graduados de psicología en Colombia, debido a que no existen estudios sobre el nivel de esta competencia en estos profesionales, lo cual representa una tarea muy importante en virtud de las competencias definidas para los profesionales de psicología en Colombia.

La Ley 1090 de 2006, que reglamenta la psicología en Colombia, indica que los psicólogos se dedican a labores investigativas, profesionales y académicas para las cuales el dominio del inglés como segunda lengua es fundamental, no solo porque la consulta y actualización teórica y empírica de las fuentes indexadas en el mundo requiere de la comprensión de textos en inglés, sino también porque la difusión de los resultados de investigación psicológica colombiana en revistas internacionales, exige que los psicólogos puedan redactar sus artículos y comunicarse con la comunidad científica en inglés (Ley 1090, 2006).

De tal manera, la Ley 1090 establece que los psicólogos se pueden dedicar a

la enseñanza, aplicación e indicación del conocimiento psicológico y de sus técnicas específicas en el diseño, ejecución y dirección de investigación científica, disciplinaria o interdisciplinaria, destinada al desarrollo, generación o aplicación del conocimiento que contribuya a la comprensión y aplicación de su objeto de estudio y a la implementación de su quehacer profesional, desde la perspectiva de las ciencias naturales y sociales (Ley 1090, 2006, p. 3).

También se especifica en dicha ley:

los profesionales de la psicología dedicados a la investigación son responsables de los temas de estudio, la metodología usada en la investigación y los materiales empleados en la misma, del análisis de sus conclusiones y resultados, así como de su divulgación y pautas para su correcta utilización (Ley 1090, 2006, p. 10). 
En los campos profesionales, el Colegio Colombiano de Psicólogos (Colpsic) ha desarrollado diferentes iniciativas para determinar las competencias del psicólogo para un ejercicio actual y futuro de la profesión en el contexto nacional e internacional, las cuales se fundamentan en los lineamiento del proyecto Tuning para Latinoamérica y el proyecto desarrollado por la International Union of Psychological Science (IUPsyS) y por la International Association of Applied Psychology (IAAP) (Colpsic, 2016). En los lineamientos de competencias genéricas del proyecto Tuning para Latinoamérica, se establece que la competencia No. 7 que debe lograrse a través de las estructuraseducativas es la comunicación en un segundo idioma. Los estudios muestran que el dominio del idioma inglés antes de ingresar a la universidad correlaciona con una gran variedad de competencias generales y ha demostrado incidir en el desarrollo de otras competencias profesionales en los egresados (Chávez-Zambrano, Saltos-Vivas \& Saltos-Dueñas, 2017; Santana, García-Santillán \& Escalen-Chávez, 2016).

En el estudio desarrollado por Rodríguez (2014), respecto de la valoración de dicha competencia en Latinoamérica, se encontró que, junto con la preservación del ambiente, tuvieron las medias más bajas en importancia.

Esta baja valoración de un segundo idioma contrasta con los lineamientos de la IUPsyS y la IAAP, en donde se establece el principio de libre circulación de los psicólogos en un mundo globalizado y para ello se requiere una comunicación fluida entre las comunidades. Es claro que la libre circulación necesita el aprendizaje y dominio del inglés, principalmente. Como señalan McDaniel, Grus, Cubic, Hunter y Kearney (2014), aún en atención primaria, los psicólogos deben poder consultar fuentes de conocimiento actuales, científicas y globales, como también desarrollar relaciones interprofesionales, para las cuales el dominio de segundo idioma es fundamental. En aquellos casos en los que el psicólogo labora en contextos internacionales, su competencia en el idioma inglés es fundamental, dada su amplia difusión en el mundo y a que se ha encontrado que la correspondencia en el idioma utilizado entre terapeuta y paciente contribuye al resultado positivo de la terapia (Bager-Charleson, Dewaele, Costa \& Kasap, 2017; Costa \& Dewaele, 2012).

De igual modo, los perfiles de los campos profesionales consignados en el documento de Colpsic (2016), incluyen en diferentes casos las competencias genéricas o transversales relacionadas con la comunicación en un segundo idioma, tal es el caso del psicólogo deportivo, como también las involucran en el perfil de desarrollo profesional en la mayoría de los campos, en los cuales se indica que el psicólogo interactúa con otras comunidades en el orden internacional; no obstante, en dichos perfiles no se hace explícita la necesidad de contar con competencias acreditadas en segundo idioma. Si bien no existen estudios particulares al contexto nacional o regional que correlacionen el dominio de una segunda lengua y los resultados en el desempeño profesional, los resultados de Colombia en cuanto a la Prueba EPI (English Proficiency Index) muestran que el país ocupa un lugar muy bajo en las puntuaciones, aun en la comparación con otros países latinoamericanos y que esta situación es generalizada a los estudiantes y egresados de diferentes campos profesionales (Sánchez, 2013). El EPI correlaciona con diferentes indicadores económicos de los países, entre los que se encuentran el empleo y la competitividad.

En segundo lugar, desde una perspectiva económica, como en otras disciplinas en nuestro país, una gran cantidad de actividades profesionales que desempeñan los psicólogos requieren el dominio del inglés, para un mejor desempeño de la función profesional. En particular, las áreas de la psicología relacionadas con la telemedicina y la gestión de los recursos humanos son dos áreas de tercerización de procesos de negocio y tercerización de procesos de conocimiento (BPO\&KPO, por sus siglas en inglés) de interés para los psicólogos, en el marco del Programa de Transformación Productiva (PTP), el cual ha identificado que uno de los sectores claves de la economía colombiana para mejorar la competitividad, aumentar el empleo y la productividad sectorial es la tercerización de procesos de negocios BPO\&KPO a diferentes regiones del mundo (Ministerio de Comercio, Industria y Turismo, s.f.).

La telemedicina es un sector en crecimiento, se espera que el mercado mundial de este servicio crezca a una tasa compuesta anual de 14,3\% entre 2014 y 2020; además, se espera que el valor del mercado alcance los 36,200 billones de dólares en 2020, más del doble del valor en 2014 (14,300 billones de dólares) (Wood, 2016). En el caso colombiano, se estimó que en 2012 los ingresos por tercerización del sector KPO (knowledge process outsourcing), telemedicina y salud fueron de 10.372 millones de pesos (IDC, 2013).

El sector de gestión de recursos humanos también es prometedor. Por ejemplo, la encuesta de Deloitte de 2014, realizada a 140 empresas entre públicas y privadas alrededor del mundo, mostró que $13 \%$ de las empresas ya utilizaban servicios de BPO de administración de recursos humanos y $38 \%$ esperaba hacerlo en el corto plazo. De igual forma, $21 \%$ de las empresas ya había contratado bajo ese medio las tareas de reclutamiento y $21 \%$ esperaba hacerlo en el corto plazo (Deloitte, 2014). En Colombia, este sector tuvo una participación del 9,2\% en el sector BPO en 2013 y generó ingresos por 685.230 millones de pesos.

En general, el análisis de los puntajes derivados de las Pruebas Saber Pro en Colombia en relación con el dominio del idioma inglés en las disciplinas en donde se ha realizado, ha mostrado que existen resultados heterogéneos en las competencias de los graduandos con respecto al tipo de institución, a la acreditación, al tamaño y a otros aspectos afines, los cuales, en conjunto, determinan el nivel de logro de las metas planteadas (Alonso et al., 2016; Alonso, Díaz, et al., 2018; Alonso, Estrada et al., 2018). El presente artículo desarrolla una descripción del cumplimiento de las metas respecto al dominio del idioma inglés $(B+)$ en los distintos programas de psicología en Colombia, y analiza las diferencias de los resultados en términos de programas de las instituciones de educación superior (IES) públicas y privadas, y la metodología del programa. Además, se presenta un análisis teniendo en cuenta los procesos de aseguramiento de la calidad establecidos como marco de la evaluación y acreditación de programas de educación superior en nuestro país (Ferreyra, Gallego \& Sarmiento, 2015).

Para tal fin, se analizan los resultados del componente en inglés del Examen de Estado de Calidad de la Educación Superior (Saber Pro) presentado por los futuros egresados de los programas de psicología de las IES entre 2011 y 2016, en los tres tipos de modalidades que existen: presencial, virtual y a distancia, y teniendo en cuenta si el programa 
se encuentra o no acreditado. $Y$ se postulan las siguientes hipótesis: (a) los programas de psicología cumplen con la meta establecida por el Gobierno colombiano, (b) todos los programas de psicología que están acreditados cumplen con la meta del Gobierno y (c) la modalidad del programa de psicología no afecta el cumplimiento de la meta.

Se eligieron los tipos de modalidad, y si está o no acreditado el programa, como variables de interés para este estudio, por varias razones. Respecto a la variable presencial, esta resulta relevante, puesto que la disponibilidad de nuevas modalidades para el desarrollo de programas (a distancia y virtual) han aumentado en los últimos años gracias a que eliminan obstáculos, como tiempo y distancia, que les impedían a las personas acceder al sistema educativo formal presencial. La modalidad virtual, o educación en línea, es un tipo de educación a distancia, que se diferencia de la primera, puesto que toda la interacción con la IES (desde la matrícula hasta el grado), con los profesores, con los compañeros de estudio y con el material se realiza virtualmente a través de las tecnologías de la información (correo, Moodle, etc.). Mientras que la educación a distancia requiere en algún momento contacto presencial en los centros de tutorías, autorizados por el MEN, donde el profesor o personal debería brindarles la atención que el estudiante requiera.

Respecto al aspecto de acreditación, este se entiende como el proceso por el cual algunos programas de pregrado deciden de manera voluntaria pasar por un proceso de evaluación de calidad por parte del Consejo Nacional de Acreditación (CNA). Este proceso tiene como objetivo producir cambios y mejorar la calidad de la educación (Ferreyra et al., 2015). Si bien el nivel de inglés de los egresados de un programa no es uno de los aspectos evaluados por el CNA, se considera que debe ser una variable de análisis, puesto que resulta interesante ver si los programas acreditados se pueden asociar con la calidad de la formación en inglés que reciben los egresados del programa.

\section{Método}

\section{Corpus de datos}

Para cumplir con los propósitos del estudio se emplearon los datos provenientes al módulo de inglés de las Pruebas Saber Pro correspondientes a 2011-2016. Prueba que es diseñada y aplicada por el Instituto Colombiano para la Evaluación de la Educación (Icfes). Este módulo de la prueba emplea el Marco Común Europeo de Referencia para las Lenguas (MCERL) que sirve como una guía estandarizada utilizada en el contexto internacional por los gobiernos, do- centes e instituciones evaluadoras para medir la competencia de quienes están aprendiendo un nuevo idioma. La tabla 2 presenta un resumen de las equivalencias de los niveles del MCERL y su adaptación a las Pruebas Saber Pro, prueba obligatoria para todos los futuros profesionales de Colombia como requisito para graduarse. La base de datos completa incluye 1.217.844 registros en total; por ejemplo: 203.815 registros para 2011 y 151.906 para 2016 (véase detalle en la tabla 3). La base de datos cuenta con 87 variables, de las cuales solo se emplean en este estudio las correspondientes a los años de toma del examen, el programa, la IES, el municipio y el desempeño en la prueba de inglés.

En la tabla 1 se muestra el número de programas que presentaron los estudiantes de psicología a la Prueba Saber Pro: 92 en 2011, 102 en 2014 y 72 en 2016. El número promedio de estudiantes por programa aumentó: de 57,3 a 105,6 desde 2011 al 2016. Para el último año, del programa de psicología de la Universidad del Valle de las sedes Buga y Palmira, 1 estudiante presentó la prueba, mientras que de la Universidad Nacional Abierta y a Distancia, 1.946 estudiantes presentaron la prueba.

Además, para determinar la acreditación del programa se emplea la base de datos construida con la información disponible en la página web del CNA, que incluye el código del programa y las fechas de inicio y finalización de la acreditación del programa. Para determinar la modalidad del programa, se emplea la base de datos del Sistema Nacional de Información de la Educación Superior (SNIES), que cuenta con el código del programa y la modalidad.

\section{Diseño}

Se realizó un estudio descriptivo retrospectivo por cohortes fijas de los resultados en las puntuaciones de inglés obtenidas en la Prueba Saber Pro, por los futuros egresados de los programas de psicología entre 2011 y 2016 en Colombia.

\section{Procedimiento}

Una vez seleccionadas las cohortes de interés, se procedió a consolidar y procesar la base de datos, así como también realizar las pruebas estadísticas a través del software R (R Core Team, 2016). El nivel de confianza usado para las conclusiones fue del $95 \%$.

Las bases de datos provenientes de la Prueba Saber Pro disponen del microdato para identificar a cada evaluado y, a su vez, discriminar de acuerdo con el programa académico,

Tabla 1. Estadísticas descriptivas de los estudiantes de psicología que toman la Prueba Saber Pro por programas (2011-2016)

\begin{tabular}{|c|c|c|c|c|c|c|}
\hline & 2011 & 2012 & 2013 & 2014 & 2015 & 2016 \\
\hline Programas que presentan estudiantes a la prueba & 92 & 93 & 96 & 102 & 109 & 72 \\
\hline Estudiantes promedio & 57,3 & 63,3 & 88,7 & 89 & 90,4 & 105,6 \\
\hline Desviación estándar & 92,9 & 114,2 & 149,9 & 160,2 & 168,4 & 227,4 \\
\hline Máximo de estudiantes & 831 & 1.030 & 1.377 & 1.494 & 1.344 & 1.946 \\
\hline Mínimo de estudiantes & 1 & 1 & 1 & 1 & 1 & 1 \\
\hline
\end{tabular}

Fuente: cálculos propios a partir de información del Icfes. 
institución educativa e información sociodemográfica, además del resultado de cada estudiante en el componente de inglés, con lo cual se puede determinar la proporción de estudiantes de psicología clasificados en nivel intermedio o superior. Para realizar este procedimiento, se seleccionan los individuos que se graduarán de programas de psicología por medio de la variable "programa", filtrándose por todos aquellos individuos que reportan psicología (véase tabla 2 para los resultados). A esta base de datos filtrada se le agrega una variable de "acreditación" que corresponde a cruzar la base de datos construida con la información disponible en la página web del CNA. Además, se crea la variable "modalidad" del programa, resultado que se cruza con la base de datos construida con la información disponible en el SNIES.

\section{Resultados}

Se analizaron los resultados para el total de evaluados de cada año en los programas de psicología del país, identificando la proporción de estos que se clasificaron en nivel $\mathrm{B}+$. Para determinar el cumplimiento de la meta de cada uno de los programas que presentaron los estudiantes de psicología a la prueba en cada uno de los años evaluados, se hicieron pruebas de proporciones paramétricas $(Z)$ y no paramétricas $\left(\chi^{2}\right)$, que permitieron contrastar la hipótesis nula que la proporción observada es menor o igual al 20\% frente a la hipótesis alterna de que dicha proporción es mayor que dicha proporción. Sin embargo, dado que se busca definir de manera simultánea si los programas de psicología cumplieron la meta en 2014, es necesario hacer un ajuste que tenga en consideración el número de comparaciones y así evitar el sesgo que se puede ocasionar al concluir resultados individuales de manera conjunta. Los ajustes se realizaron empleando los métodos sugeridos por Holm (1979), Hochberg (1966), Hommel (1988), Benjamini y Hochberg (1995) (BH), Yekutieli y Benjamini (1999) (BY) y Bonferroni (1936). De esta manera, la decisión respecto al cumplimiento o no de la meta, se tomó con base en la mayoría de los criterios.

En 2016, el 5\% de los estudiantes evaluados pertenecían a un programa de psicología; de estos, 7,4\% alcanzaron un nivel intermedio o superior en inglés, mientras que $11,2 \%$ del total de programas relacionados con las ciencias sociales y humanas (según SNIES, programas que pertenecen al área de conocimiento: ciencias sociales y humanas) alcanzaron $\mathrm{B}+$. Este resultado fue inferior al de los programas de medicina, en los cuales $24,2 \%$ de sus estudiantes clasificó en nivel $\mathrm{B}+$, mientras que todas las ciencias de la salud (según SNIES, programas que pertenecen al área de conocimiento: ciencias de la salud) el 9,4\% de estudiantes alcanzaron este nivel. Los programas de administración de empresas clasifican para el mismo año al 8,6\% de sus estudiantes en nivel intermedio o superior, mientras que el total de programas del área de conocimiento de economía, administración, contaduría y afines (según SNIES, programas que pertenecen al área de conocimiento: economía, administración, contaduría y afines) clasifican $8,9 \%$ y esta cifra es de $12,5 \%$ para los programas de ingeniería (según SNIES, programas que pertenecen al área de conocimiento: ingeniería, arquitectura, urbanismo y afines.). El número de evaluados en psicología en 2011 fue de 5.275 estudiantes, este número creció hasta 2015 y cerró en 2016 con 7.602 estudiantes, lo cual representa un crecimiento del $44 \%$. Asimismo, más del $70 \%$ de los estudiantes de psicología que presentaron la prueba, pertenecen a programas de modalidad presencial y clasifican mayor proporción de estudiantes en $\mathrm{B}+$ que las modalidades virtual y a distancia, para todos los años de estudio (véase tabla 3).

De la base de datos del Consejo Nacional de Acreditación (CNA), se encuentra que de los 92 programas que presentaron los estudiantes de psicología a la prueba en 2011, 26 estaban acreditados y 66 no lo estaban. En 2014, 32 programas de psicología estaban acreditados y 70 no tenían acreditación. En los dos últimos años, el número de programas acreditados con estudiantes que tomaron la prueba fue de 33 y 25 para 2015 y 2016, respectivamente.

En términos generales, el $8,9 \%$ de los estudiantes próximos a graduarse de psicología obtuvieron una clasificación de $\mathrm{B}+$ en el módulo de inglés de Saber Pro de 2014. Este no fue mayor significativamente al $20 \%$, por lo que en ese año no se cumplió la meta. Dicho porcentaje no aumenta en 2015 (8,9\%) e incluso disminuye en 2016 (7,4\%). Ahora bien, cuando se analiza por modalidad de enseñanza, se encuentra que los estudiantes de la modalidad presencial obtuvieron un valor similar al general, pero los estudiantes de modalidad a distancia y virtual presentan un valor mucho menor de $3,6 \%$ y $3,3 \%$, respectivamente. Lo anterior ocurre para 2014 y se mantiene de esa forma hasta 2016.

La tabla 4 muestra un escalafón de los 20 programas con mayor proporción de estudiantes clasificados en nivel intermedio o superior en 2016. En ella se presenta la posición en el escalafón desde 2011 al 2016, la institución y municipio donde se ofrece el programa, así como también

Tabla 2. Equivalencias entre los niveles de referencia del MCERL, Nacional y clasificación de la Prueba Saber Pro

\begin{tabular}{|c|c|c|c|}
\hline Clasificación del usuario & Nivel MCERL & Equivalencia en Colombia & Nivel en Saber Pro \\
\hline & & & $A-$ \\
\hline \multirow{2}{*}{ Usuario básico } & $\mathrm{A} 1$ & Principiante & $\mathrm{A} 1$ \\
\hline & $\mathrm{A} 2$ & Básico & $\mathrm{A} 2$ \\
\hline \multirow{2}{*}{ Usuario independiente } & B1 & Pre-intermedio & B1 \\
\hline & B2 & Intermedio & \\
\hline \multirow{2}{*}{ Usuario competente } & $\mathrm{C} 1$ & Pre-avanzado & $\mathrm{B}+$ \\
\hline & $\mathrm{C} 2$ & Avanzado & \\
\hline
\end{tabular}

Fuente: adaptada a partir de Alonso et al. (2012). 
Tabla 3. Porcentaje de estudiantes en nivel intermedio o superior $(B+)$ de acuerdo con la Prueba Saber Pro por programa (2011-2016)

\begin{tabular}{lcccccccccccc}
\hline & \multicolumn{2}{c}{2011} & \multicolumn{2}{c}{2012} & \multicolumn{2}{c}{2013} & \multicolumn{2}{c}{2014} & \multicolumn{2}{c}{2015} & \multicolumn{2}{c}{2016} \\
\hline Programas & $\mathrm{N}^{*}$ & $\% \mathrm{~B}+$ & $\mathrm{N}^{*}$ & $\% \mathrm{~B}+$ & $\mathrm{N}^{*}$ & $\% \mathrm{~B}+$ & $\mathrm{N}^{*}$ & $\% \mathrm{~B}+$ & $\mathrm{N}^{*}$ & $\% \mathrm{~B}+$ & $\mathrm{N}^{*}$ & $\% \mathrm{~B}+$ \\
\hline $\begin{array}{l}\text { Ciencias sociales } \\
\text { y humanas }\end{array}$ & 44.018 & $6,5 \%$ & 42.345 & $8,1 \%$ & 40.954 & $10,7 \%$ & 41.637 & $11,6 \%$ & 49.530 & $10,8 \%$ & 34.012 & $11,2 \%$ \\
Psicología & 5.275 & $6,5 \%$ & 5.861 & $7,4 \%$ & 8.519 & $7,9 \%$ & 9.083 & $8,9 \%$ & 9.859 & $8,9 \%$ & 7.602 & $7,4 \%$ \\
Distancia & 1.031 & $1,3 \%$ & 1.226 & $1,1 \%$ & 1.599 & $1,1 \%$ & 2.249 & $1,5 \%$ & 2.622 & $1,4 \%$ & 2.126 & $0,4 \%$ \\
Presencial & 4.197 & $7,8 \%$ & 4.595 & $9,1 \%$ & 6.877 & $9,6 \%$ & 6.737 & $11,4 \%$ & 6.970 & $12,0 \%$ & 5.476 & $10,1 \%$ \\
Virtual & 47 & $2,1 \%$ & 40 & $2,5 \%$ & 43 & $4,7 \%$ & 97 & $3,1 \%$ & 267 & $4,5 \%$ & 0 & $\mathrm{NA}$ \\
Ciencias de la salud & 17.950 & $6,0 \%$ & 18.157 & $8,4 \%$ & 23.371 & $7,8 \%$ & 19.638 & $9,2 \%$ & 21.749 & $10,4 \%$ & 14.965 & $9,4 \%$ \\
Medicina & 4.735 & $17,2 \%$ & 5.087 & $23,4 \%$ & 6.673 & $20,8 \%$ & 5.349 & $25,2 \%$ & 6.099 & $28,4 \%$ & 4.814 & $24,2 \%$ \\
$\begin{array}{l}\text { Economía, } \\
\text { administración, }\end{array}$ contaduría y afines & 57.795 & $5,6 \%$ & 65.588 & $6,5 \%$ & 68.229 & $7,7 \%$ & 64.867 & $7,9 \%$ & 73.462 & $7,8 \%$ & 36.186 & $8,9 \%$ \\
$\begin{array}{l}\text { Administración de } \\
\text { empresas }\end{array}$ & 16.358 & $7,4 \%$ & 18.360 & $8,2 \%$ & 24.004 & $8,5 \%$ & 21.877 & $9,1 \%$ & 23.253 & $9,3 \%$ & 12.951 & $8,6 \%$ \\
$\begin{array}{l}\text { Ingeniería } \\
\text { Resto }\end{array}$ & 43.300 & $9,6 \%$ & 46.080 & $11,8 \%$ & 50.481 & $14,0 \%$ & 43.399 & $15,9 \%$ & 50.011 & $15,8 \%$ & 38.219 & $12,5 \%$ \\
$\begin{array}{l}\text { Todos los } \\
\text { programas }\end{array}$ & 40.752 & $8,2 \%$ & 38.105 & $11,2 \%$ & 47.911 & $11,9 \%$ & 43.569 & $12,1 \%$ & 43.040 & $14,0 \%$ & 28.524 & $11,8 \%$ \\
\hline
\end{tabular}

Nota. Los programas ciencias sociales y humanas y psicología, no son mutuamente excluyentes; es decir, que el número de estudiantes que se encuentran en psicología también forma parte del primer grupo. Lo mismo aplica para ciencias de la salud y medicina; economía, administración, contaduría y afines y administración de empresas.

Fuente: cálculos propios a partir de información del Icfes.

la proporción de estudiantes que se clasificaron en cada nivel durante 2016 y el número total de estudiantes. En esta tabla se pueden resaltar cuatro puntos. Primero, no existe en Colombia un programa de psicología que clasifique el $100 \%$ de sus estudiantes en nivel B+. Segundo, solo 13 programas de 72 clasifican al menos al 20\% de estudiantes en nivel intermedio o superior (sin realizar pruebas estadísticas). Tercero, los programas de psicología incluidos en este escalafón pertenecen a instituciones ubicadas en los departamentos de Cundinamarca, Antioquia, Atlántico, Santander, Valle, Bolívar, Risaralda, Tolima y Boyacá; 14 de ellos se concentran en las ciudades de Bogotá, Medellín y Cali. Y cuarto, todos los programas que clasifican al menos el $20 \%$ de sus estudiantes en $\mathrm{B}+$, se ubicaron dentro de los primeros puestos en los años anteriores. Cabe aclarar que si bien 13 programas clasifican al menos el $20 \%$ de estudiantes en $\mathrm{B}+$, luego se realizarán las pruebas estadísticas correspondientes para determinar el cumplimiento o no de la meta establecida.

La figura 1 muestra los resultados de los valores $p$ de las pruebas paramétricas y no paramétricas para cada ajuste empleado. La figura representa el tipo de ajuste utilizado, y el color representa si el programa estaba acreditado o no (gris para los acreditados y en negro para los no acreditados).

Concentrando la atención en 2014, año para el que se fijó la meta, se encuentra que de los 102 programas activos en ese año, 26 no tuvieron ningún estudiante en $\mathrm{B}+\mathrm{y}$, por ende, solo se realiza la prueba de proporciones no paramétrica y paramétrica para los 76 programas restantes, de los cuales 72 son presenciales, tres a distancia y uno virtual.

Por otra parte, bajo ambas pruebas, paramétrica y no paramétrica, se encuentra que solo seis programas (el $6 \%$ del total de programas) cumplieron con la meta (con un $p$ valor cercano a cero): estos programas pertenecen de las siguientes IES: Colegio Mayor de Nuestra Señora del Rosario, Fundación Universidad del Norte, Pontificia Universidad Javeriana - Bogotá, Universidad de la Sabana, Universidad de los Andes y Universidad Nacional de Colombia. Todos estos son presenciales y cuatro de ellos estuvieron acreditados durante 2014. Esos programas corresponden a las siguientes IES (ordenadas alfabéticamente): Colegio Mayor de Nuestra Señora del Rosario $\left(\chi^{2}(31, n=32)=50.63, p=.00\right)$, Fundación Universidad del Norte $\left(\chi^{2}(141, n=142)=34.75, p=.00\right)$, Pontificia Universidad Javeriana $\left(\chi^{2}(148, n=149)=170.21, p=.00\right)$, Universidad de La Sabana $\left(\chi^{2}(140, n=141)=116.65, p=.00\right)$, Universidad de los Andes $\left(\chi^{2}(126, n=127)=300.18, p=.00\right)$, Universidad Nacional de Colombia $\left(\chi^{2}(101, n=102)=63.14, p=.00\right)$. Esta última es la única universidad, dentro del subconjunto de seis universidades, que pertenece al sector público. Además de eso, se observa que todos los programas que superaron la meta se encuentran ubicados en Bogotá, con excepción del de la Fundación Universidad del Norte, cuya sede se encuentra en Barranquilla.

Asimismo, en 2015, según la aproximación paramétrica, nueve de 109 programas cumplieron con la meta (8\%), todos son presenciales, y seis de ellos estuvieron acreditados durante 2015. Esos programas corresponden a las mismas IES que cumplieron la meta en 2014, además del Instituto de Ciencias de la Salud $(Z(n=38)=6.56, p=.00)$, de la Universidad Eafit $(Z(n=20)=3.27, p=.04)$ y Universidad Pontificia Bolivariana $(Z(n=63)=3.56, p=.003)$. También se observa 


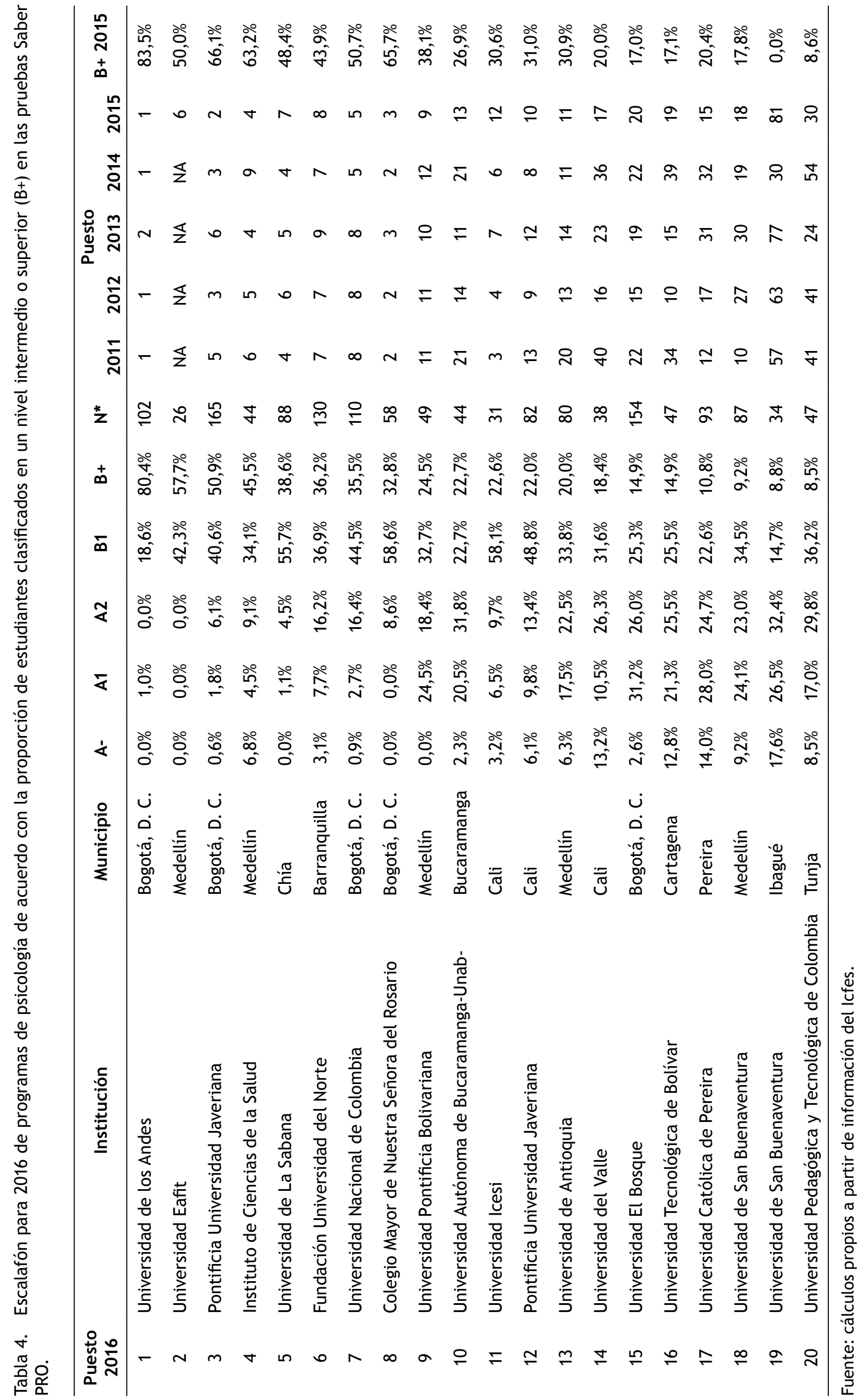


Corporación Universidad Piloto De Colombia-Bogotá D.C. Corporación Universitaria Del Caribe-SincelejoCorporación Universitaria Minuto De Dios-Bello Fundación Universitaria De Ciencias De La Salud-Bogotá D.C. Fundación Universitaria Konrad Lorenz-Bogotá D.C. -
Fundación Universitaria Los Libertadores-Bogotá D.C. Fundación Universitaria Luis Amigo Funlam-Bogotá D.C. -

Fundación Universitaria Luis Amigo Funlam-Medellin Fundación Universitaria María Cano-Medellín -

Fundación Universitaria San Martin-Bogotá D.C. Fundación Universitaria Sanitas-Bogotá D.C. Institución Universitaria De Envigado-EnvigadoInstituto De Ciencias De La Salud-Medellin Politécnico Grancolombiano-Bogotá D.C. Pontificia Universidad Javeriana-Cali Uniminuto- Corporación Universitaria Minuto De Dios-Bogotá D.C. Universidad Autónoma De Bucaramanga-Unab--Armenia Universidad Autónoma De Bucaramanga-Unab--Bucaramanga Universidad Autónoma Del Caribe-BarranquillaUniversidad Católica De Colombia-Bogotá D.C. -
Universidad Católica De Oriente-RionegroUniversidad Católica Popular Del Risaralda-Pereira Universidad Cooperativa De Colombia-Barrancabermeja -

Universidad Cooperativa De Colombia-Bogotá D.C. Universidad Cooperativa De Colombia-Bucaramanga Universidad Cooperativa De Colombia-Cali Universidad Cooperativa De Colombia-Neiva -

Universidad Cooperativa De Colombia-Popayán Universidad Cooperativa De Colombia-VillavicencioUniversidad De Antioquia-Carmen de ViboralUniversidad De Antioquia-Medellin Universidad De Antioquia-TurboUniversidad De Antioquia-Yarumal -
Universidad De Ibagué -Coruniversitaria--Ibagué -
Universidad De Manizales-Manizales Universidad De Pamplona-Pamplona Universidad De San Buenaventura-ArmeniaUniversidad De San Buenaventura-Bogotá D.C. Universidad De San Buenaventura-Cali Universidad De San Buenaventura-CartagenaUniversidad De San Buenaventura-lbagué Universidad De San Buenaventura-Medellín Universidad Del Magdalena-Santa Marta Universidad Del Sinú Elías Bechara Zainum - Unisinú--Cartagena Universidad Del Sinú Elías Bechara Zainum - Unisinú--Monteria Universidad Del Valle-Cali -
Universidad Del Valle-Guadalajara De Buga-
Universidad Del Valle-PalmiraUniversidad Del Valle-Palmira -
rsidad EI Bosque-Bogotá D.C. Universidad El Bosque-Bogota D.C. -
Universidad Icesi-Cali -

Universidad Incca De Colombia-Bogotá D.C. Universidad Libre-Cali Universidad Manuela Beltran-Umb--Bogotá D.C. -
Universidad Mariana-Pasto Universidad Metropolitana-Barranquilla Universidad Pedagógica Y Tecnológica De Colombia-Tunja Universidad Pontificia Bolivariana-Bucaramanga Universidad Pontificia Bolivariana-Medellin Universidad Pontificia Bolivariana-Medellin Universidad Pontificia Bolivariana-Monteria -
Universidad Pontificia Bolivariana-Palmira Universidad Santiago De Cali-Cali Universidad Santo Tomas-Bogotá D.C. Universidad Simón Bolivar-Barranquilla Universidad Simón Bolívar-Cúcuta Universidad Surcolombiana-Neiva Universidad Tecnológica De Bolivar-Cartagena -

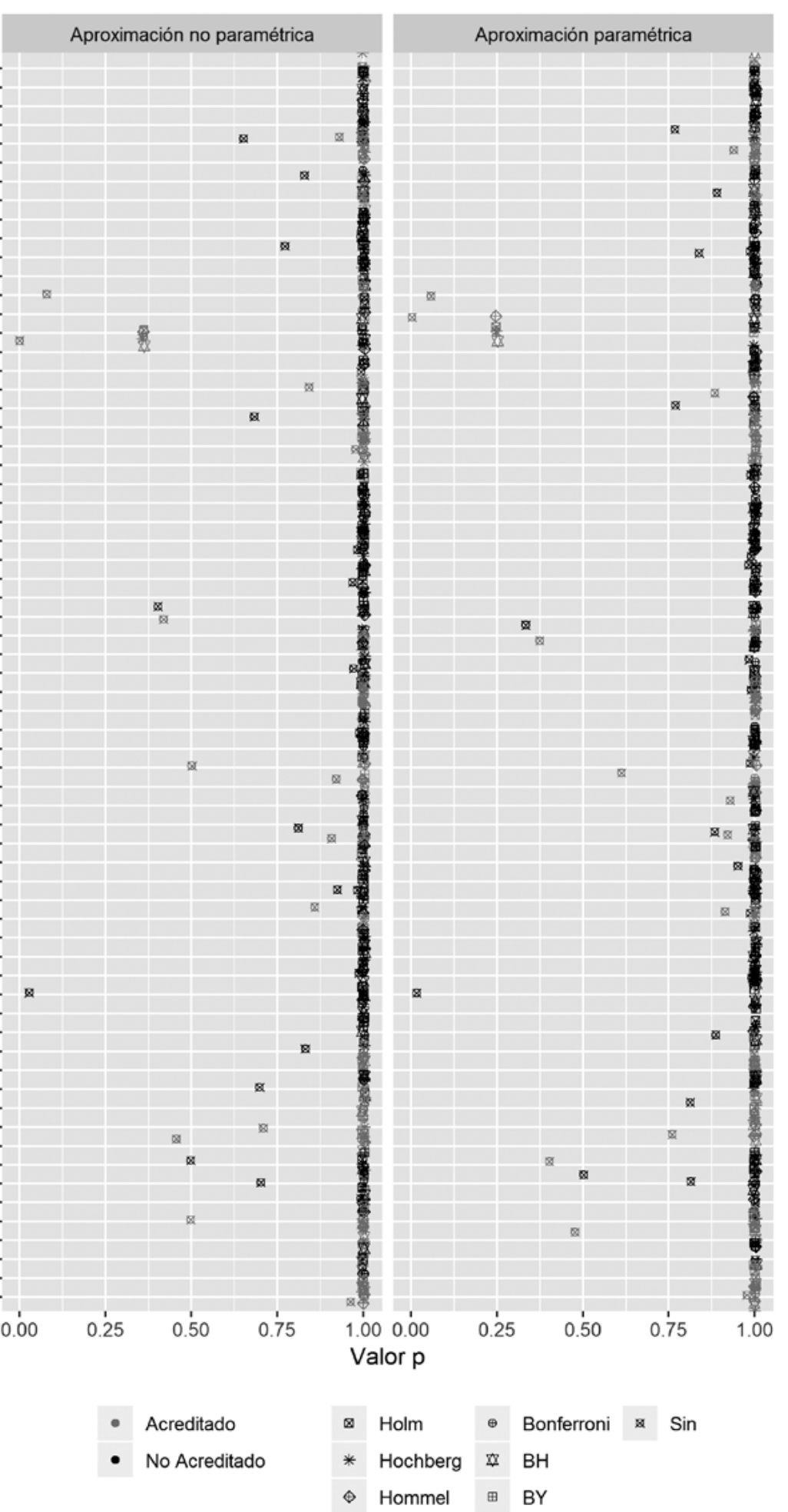

Figura 1. Programas de modalidad presencial que no cumplen con la meta: aproximación paramétrica y no paramétrica (2014) 
que $56 \%$ de las universidades que superaron la meta se encuentran ubicadas en Bogotá, con excepción de la Fundación Universidad del Norte, cuya sede se encuentra en Barranquilla, del Instituto de Ciencias de la Salud y de la Universidad Eafit, ubicadas en Medellín. Estos resultados se mantienen en la aproximación no paramétrica a excepción de la Universidad Eafit $\left(\chi^{2}(101, N=102)=9.45, p=.11\right)$, quien no cumple la meta según esta aproximación (todos los demás $p$ valores son cercanos a cero). Por razones de espacio no se presentan los resultados de esta prueba para estos programas; sin embargo, estos se encuentran disponibles para el lector que los desee solicitar (Los resultados se pueden solicitar al autor para correspondencia).

Por otra parte, los $p$ valores para las pruebas de los otros programas presenciales (que no cumplen con la meta), se muestran en orden alfabético en la figura 1. Los programas bajo esta modalidad que presentan estudiantes a la Prueba Saber Pro en 2014, para los cuales no se puede rechazar la hipótesis nula en al menos cuatro de los siete criterios y que, por ende, no alcanzan a cumplir la meta son 66; esto corresponde al $92 \%$ de los programas presenciales; asimismo, 26 (39\%) de estos son programas acreditados. Estos resultados se mantienen empleando tanto la prueba paramétrica como la no paramétrica.

En cuanto al cumplimiento de las metas por parte de los programas acreditados, solo seis de los 102 programas superaron la meta en 2014 y cuatro de estos programas eran acreditados. Además, se observó que 62 programas no lograron clasificar en B+ más del $5 \%$ de sus estudiantes en 2014 y 11 de estos se encontraban acreditados. Para finalizar, según la tabla 5, no se observa una tendencia de mejora en 2015 y 2016, pues los programas que cumplen son los mismos que en 2014 con unas pocas excepciones.

El número de programas que presentan los estudiantes a la Prueba Saber Pro en 2014 bajo la modalidad a distancia son tres, mientras que bajo la modalidad virtual existe solo un programa. Se encuentra que no se puede rechazar la hipótesis nula para ninguno de los tres programas a distancia y, además, el programa virtual no clasifica a ninguno de sus estudiantes en $\mathrm{B}+(\mathrm{el} p$ valor es de 1$)$, sin embargo, por razones de espacio no se presentan los resultados de esta prueba, pero estos se encuentran disponibles al lector que los quiera solicitar. Luego, el $100 \%$ de los programas de psicología de modalidades a distancia y virtual no cumplieron con la meta establecida por el Proyecto de Fortalecimiento al Desarrollo de Competencias en Lenguas Extranjeras (PFDCLE). Estos resultados se mantienen empleando la prueba paramétrica. Resultado similar se presenta para los otros años evaluados. Es importante resaltar que no hay programas de psicología acreditados de modalidad virtual y distancia.

\section{Discusión}

Los resultados presentados muestran que los programas de psicología colombianos en su mayoría no cumplieron con la meta del $20 \%$ en B+ propuesta por el Gobierno Nacional para 2014, de acuerdo con las pruebas paramétricas y no paramétricas. Este resultado es independiente de la modalidad de enseñanza (presencial, a distancia o virtual). Es más, solo 6 de los 102 programas presenciales cumplieron la meta en 2014, y ninguno de los programas a distancia o virtual alcanzó la meta. De estos seis programas que sí cumplieron con la meta, uno pertenece a una IES oficial y el resto a IES privadas; además, cinco de estos se encuentran ubicados en Bogotá.

De acuerdo con los criterios establecidos para la formación en psicología por el proyecto Tuning para Latinoamérica y por el proyecto desarrollado por la IUPsyS (International Union of Psychological Science) y por la IAAP (International Association of Applied Psychology) (Colpsic, 2016), el presente estudio demuestra que la competencia de comunicación en un segundo idioma no se ha logrado en Colombia y que los procesos de apoyo al bilingüismo en las instituciones universitarias no están generando los resultados esperados. Esto podría significar que el modelo pedagógico que sustenta estas estrategias debe ajustarse hacia las competencias de entrada y que es necesario incorporar los hallazgos en el campo del aprendizaje de segunda lengua en adultos, creando un modelo apropiado tanto a la disciplina como a las características de los estudiantes de psicología (Adesope, Lavin, Thompson y Ungerleider, 2010; Antoniou, Liang, Ettlinger y Wong, 2015; Bialystok, Craik y Luk, 2008).

Desde la perspectiva institucional, los resultados hacen evidente la necesidad de alinear las metas de política públi-

Tabla 5. Resumen. Cumplimiento de meta de tener más del $20 \%$ de estudiantes en clasificación B+ en al menos un período: IES acreditadas en 2014 de psicología, modalidad presencial (2011-2016) (orden alfabético de las universidades)

\begin{tabular}{|c|c|c|c|c|c|c|c|}
\hline Institución & $\begin{array}{c}\text { Municipio de } \\
\text { oferta }\end{array}$ & 2011 & 2012 & 2013 & 2014 & 2015 & 2016 \\
\hline Fundación Universidad del Norte & Barranquilla & $\checkmark$ & $x$ & $x$ & $\checkmark$ & $\checkmark$ & $\checkmark$ \\
\hline Instituto de Ciencias de la Salud & Medellín & $x$ & $\checkmark$ & $\checkmark$ & $x$ & $\checkmark$ & $\checkmark$ \\
\hline Pontificia Universidad Javeriana & Bogotá, D. C. & $\checkmark$ & $\checkmark$ & $\checkmark$ & $\checkmark$ & $\checkmark$ & $\checkmark$ \\
\hline Universidad de La Sabana & Chía & $\checkmark$ & $\checkmark$ & $\checkmark$ & $\checkmark$ & $\checkmark$ & $\checkmark$ \\
\hline Universidad Nacional de Colombia & Bogotá, D. C. & $x$ & $x$ & $\checkmark$ & $\checkmark$ & $\checkmark$ & $\checkmark$ \\
\hline Universidad Pontificia Bolivariana & Medellín & $x$ & $x$ & $x$ & $x$ & $\checkmark$ & $x$ \\
\hline
\end{tabular}

Nota: $\checkmark$ IES cumplió la meta, al rechazar la hipótesis nula (proporción menor o igual a 0,2) en favor de la alternativa (proporción mayor a 0,2 ) con $95 \%$ de confianza. En caso de que la decisión no sea unánime bajo los seis criterios para evaluar el valor $p$, se elige lo que indique la mayoría de los criterios. × IES no cumplió la meta (no se rechaza la hipótesis nula).

Fuente: cálculos propios a partir de información del Icfes. 
ca y las acciones de las IES privadas y públicas para lograr aumentar el nivel de dominio del inglés de los futuros psicólogos y demás profesionales colombianos.

Desde el punto de vista de la política pública, estos resultados urgen a revisar y evaluar el impacto de las acciones llevadas a cabo en el PFDCLE, y las que se están realizando en el Programa Nacional de Inglés 2015-2025 Colombia Very Well! Por consiguiente, es necesario monitorear para retroalimentar y mejorar el proceso de enseñanza del inglés a través de acciones de política pública, como también es necesario revisar la forma en que se está articulando la enseñanza del inglés entre la educación secundaria y la educación superior. Esto es relevante, puesto que este no es el primer intento del Ministerio de Educación por mejorar los niveles de inglés de la población estudiantil mediante políticas públicas. En 1987 lanzó el programa “Currículo de Inglés", que introdujo un currículo estandarizado de inglés para los grados sexto, noveno, décimo y once, y la obligatoriedad de recibir inglés en sexto y séptimo; posteriormente, lanzó el proyecto “Colombian Framework for English" (COFE), que se ejecutó entre 1991 y 1996 con el fin de incrementar el nivel de inglés de los profesores; finalmente, en 2004 empezó el Programa Nacional de Bilingüismo cuya duración abarcaba de 2004 al 2019. Sin embargo y a diferencia de los proyectos anteriores, este es el primer programa que se propuso unas metas intermedias específicas, definidas en el tiempo y medibles.

Por otro lado, de los seis programas de modalidad presencial que sí cumplieron con la meta establecida por el PFDCLE en 2014, cuatro de estos se encontraban acreditados. No obstante, esta cifra también es preocupante, porque los otros 26 programas acreditados no cumplieron la meta. Es importante que este análisis se extienda a otras disciplinas y se valide si es un problema generalizado, de ser así se podría evaluar la calidad del inglés que reciben los estudiantes en el componente al proceso de acreditación (no solo su existencia) y evaluar el impacto que esto tiene en el desempeño de los estudiantes. Asimismo, debe revisarse la política pública del Ministerio de Comercio, Industria y Turismo en torno al Programa de Transformación Productiva (PTP) y cómo esta se puede articular de una mejor manera a la política de bilingüismo del país elaborada por el Ministerio de Educación.

Finalmente, es importante reconocer que este análisis no da cuenta del valor agregado por las IES en la competencia en inglés en estos programas. Tampoco estudia cuál es el nivel de inglés en otras competencias como comprensión auditiva, expresión oral o expresión escrita, competencias que podrían ser más valiosas para los psicólogos en su profesión que la comprensión de lectura. Estos aspectos serán hipótesis interesantes a explorar en futuras investigaciones.

\section{Referencias}

Adesope, O., Lavin, T., Thompson, T., \& Ungerleider, Ch. (2010). A systematic review and meta-analysis of the cognitive correlates of bilingualism. Review of Educational Research, 80(2), 207-245. http://dx.doi.org/10.3102/0034654310368803

Alonso, J. C., Casasbuenas, P., Gallo, B., \& Torres, G. (2012). Bilingüismo en Santiago de Cali: análisis de los resultados de las Pruebas SABER 11 y SABER PRO. Cali: Universidad Icesi.

Alonso, J. C., Díaz, D. M., Estrada, D., \& Mueces, B. V. (2018). Nivel de inglés de los futuros egresados de los programas de pregrado de medicina en Colombia. Revista de la Facultad de Medicina, 66(2), 459-466. http://dx.doi.org/10.15446/revfacmed.v66n2.61296

Alonso, J. C., Estrada, D., \& Mueces, B. V. (2018). Nivel de inglés en los programas de economía de Colombia: ¿se cumple la meta? Lectura de Economía, 89, 41-67.

Alonso, J. C., Estrada, D., \& Martínez, D. A. (2016). ¿Se cumplió la meta de bilingüismo en los programas de educación universitaria del sector software en Colombia? Revista Educación en Ingeniería, 12(22), 39-45. http://dx.doi.org/10.26507/rei. v11n22.649

Antoniou, M., Liang, E., Ettlinger, M., \& Wong, P. C. M. (2015). The bilingual advantage in phonetic learning. Bilingualism: Language and Cognition, 18(04), 683-695. http://dx.doi. org/10.1017/s1366728914000777

Bager-Charleson, S., Dewaele, J.-M. D., Costa, B., \& Kasap, Z. (2017). A multilingual outlook: Can awareness-raising about multilingualism affect therapists' practice? A mixed-method evaluation. Language and Psychoanalysis, 6(2), 56-75. http:// dx.doi.org/10.7565/landp.v6i2.1572

Benjamini, Y., \& Hochberg, Y. (1995). Controlling the false discovery rate: a practical and powerful approach to multiple testing. Journal of the Royal Statistical Society. Series B (Methodological), 57(1), 289-300.

Bialystok, E., Craik, F. I. M., \& Luk, G. (2008). Lexical access in bilinguals: Effects of vocabulary size and executive control. Journal of Neurolinguistics, 21(6), 522-538. http://dx.doi. org/10.1016/j.jneuroling.2007.07.001

Bonferroni, C. E. (1936). Teoria statistica delle classi e calcolo delle probabilità. Pubblicazioni del $R$ Istituto Superiore di Scienze Economiche e Commerciali di Firenze, 8, 3-62.

Chávez-Zambrano, M., Saltos-Vivas, M., \& Saltos-Dueñas, C. (2017). La importancia del aprendizaje del idioma inglés en la enseñanza superior. Dominio de las Ciencias, 3, 759-771.

Colegio Colombiano de Psicólogos (Colpsic). (2016). Perfiles por competencias del profesional en psicología. Bogotá. Recuperado de https://issuu.com/colpsic/docs/perfiles_por_competencia_del_profes.

Congreso de Colombia. (6, septiembre, 2016). Reglamentación del ejercicio de la profesión de Psicología y dictación del Código Deontológico y Bioético y otras disposiciones [Ley 1090 de 2006]. Recuperado de http://www.sociedadescientificas.com/ userfiles/file/LEYES/1090\%2006.pdf.

Costa, B., \& Dewaele, J. M. (2012). Psychotherapy across languages: Beliefs, attitudes and practices of monolingual and multilingual therapists with their multilingual patients. Language and Psychoanalysis. 1, 18-40. http://dx.doi.org/10.7565/ landp./2012.0003

Deloitte. (2014). Deloitte's 2014 Global Outsourcing and Insourcing Survey. Recuperado de https://www2.deloitte.com/content/ dam/Deloitte/us/Documents/strategy/us-2014-global-outsourcing-insourcing-survey-report-123114.pdf.

Ferreyra, H. A., Gallego, J. D., \& de Sarmiento, M. O. (2015). La evaluación institucional de la educación superior en Colombia: aproximación a un estado de la cuestión. Revista Latinoamericana de Políticas y Administración de la Educación, 2(2), 138-148.

Fidrmuc, J., \& Fidrmuc, J. (2014). Foreign languages and trade. CESifo Working Paper Series No. 4670.

Hochberg, Yosef (1966). A sharper Bonferroni procedure for multiple tests of significance. Biometrika, 75(4), 800-802.

Holm, Sture (1979). A simple sequentially rejective multiple test procedure. Scandinavian Journal of Statistics, 6(2), 65-70.

Hommel, Gerhard (1998). A stagewise rejective multiple test procedure based on a modified Bonferroni test. Biometrika, 75(2) 383- 386.

International Data Corporation (IDC). (2013). Caracterización y formulación estratégica del sector BPO, KPO e ITO en Colombia. Resumen Ejecutivo. Recuperado de https://www.ptp.com.co/ documentos/5\%20IDC_PTP_Resumen\%20ejecutivo\%20final_Pu- 
blicado_II.pdf.

Ku, H., \& Zussman, A. (2010). Lingua franca: The role of English in international trade. Journal of Economic Behavior \& Organization, 75(2), 250-260. http://dx.doi.org/10.1016/j. jebo.2010.03.013

Lee, C. G. (2012). English language and economic growth: Crosscountry empirical evidence. Journal of Economic and Social Studies, 2(1), 5-20. http://dx.doi.org/10.14706/jecoss11211

McDaniel, S. H., Grus, C., Cubic, B., Hunter, C., Kearney, L., Schuman, C. C., Karel, M. J. ... Bennett Johnson, S. (2014). Competencies for psychology practice in primary care. American Psychologist, 69(4), 409-429.

Ministerio de Educación Nacional (MEN). (2009). Educación virtual o educación en línea. Recuperado de https://www.mineducacion.gov.co/1759/w3-article-196492.html.

Ministerio de Educación Nacional (MEN). (2014). Programa Nacional de Inglés 2015-2025. Recuperado de http://www.colombiaaprende.edu.co/html/micrositios/1752/articles-343287_recurso_1.pdf.

Ministerio de Educación Nacional (MEN). (2015). Seguimiento a las condiciones de vinculación laboral y resultados de la encuesta de percepción de los recién graduados. Bogotá. Recuperado de https://www.mineducacion.gov.co/1759/articles-355258_archivo_pdf.pdf.

Ministerio de Comercio, Industria y Turismo. (s.f.). Programa de Transformación Productiva. Disponible en https://www.ptp. com.co/contenido/contenido. aspx?catID=607\&conID=1.

R Core Team. (2016). R: A language and environment for statistical computing [software]. R Foundation for Statistical Computing.
Vienna, Austria. Disponible en https://www.R-project.org/.

Rodríguez, D. E. (2014). Meta-perfil del área de Psicología. En Pablo, B., González, J., \& Wagenaar, R. (Eds.). Meta-perfiles y perfiles: una nueva aproximación para las titulaciones en América Latina. Universidad de Deusto (España), Proyecto Tuninig. Recuperado de http://tuning.unideusto.org/tuningal/index. php?option=content\&task=view\&id $=172 \&$ Itemid $=200$.

Sánchez, A. (2013). Bilingüismo en Colombia. Centro de Estudios Regionales del Banco de la República, Cartagena (Colombia). Recuperado de http://www.banrep.gov.co/docum/Lectura_finanzas/pdf/dtser_191.pdf.

Santana, J., García-Santillán, A., \& Escalen-Chávez, M. (2016). Variables que influyen sobre el aprendizaje del inglés como segunda lengua. Revista Internacional de Lenguas Extranjeras, 5, 79-94. http://dx.doi.org/10.17345/rile201679-94

Wood, M. (5, octubre, 2016). Telemedicine to attract 7M patient users by 2018 - 12 statistics on the thriving market. Beckers Hospital Review. Recuperado de https://www.beckershospitalreview.com/healthcare-information-technology/telemedicine-to-attract-7m-patient-users-by-2018-12-statistics-on-thethriving-market.html.

Yekutieli, Daniel \& Benjamini, Yoav (1999). Resampling-based false discovery rate controlling multiple test procedures for correlated test statistics. Journal of Statistical Planning and Inference. 82(1), 171-96. 